\title{
Study on The Effect Mechanism of "Common Treatment for Different Diseases" of Dachaihu Decoction on Prediabetes and Acute Hemorrhagic Stroke Based on Network Pharmacology
}

\author{
Xiaolin Zhang \\ Changchun University of Chinese Medicine \\ Di Cao \\ Changchun hospital of chinese medicine \\ Qi Zhang \\ Changchun University of Chinese Medicine \\ Dehui Ma \\ Changchun University of Chinese Medicine \\ Mingjun Liu ( $\square$ mingjunliu646590@163.com ) \\ Changchun University of Chinese Medicine
}

\section{Research}

Keywords: Dachaihu Decoction, Network Pharmacology, Prediabetes, Acute hemorrhagic stroke, Common treatment for different diseases

Posted Date: June 30th, 2020

DOI: https://doi.org/10.21203/rs.3.rs-37227/v1

License: (c) (i) This work is licensed under a Creative Commons Attribution 4.0 International License.

Read Full License 


\section{Abstract}

Background: In this study, network pharmacology method was used to systematically predict and analyze the mechanism of "Common treatment for different diseases" effect of Dachaihu Decoction(DCHD) in the treatment of Prediabetes(PD) and Acute hemorrhagic stroke(AHS).

Methods: TCMsp (Traditional Chinese Medicine systems pharmacology database and analysis platform) database was used to collect all the candidate active components related to 8 kinds of traditional Chinese medicine of DCHD, and UniProt database was used to obtain the drug action target and construct the "traditional Chinese medicine -Compound -target" action network; Genecards, OMIM(Online Mendelian Inheritance in Man), DisGeNET, CTD(Comparative Toxicogenomics Database) and TTD $\triangle$ Therapeutic Target Database $\llbracket$ databases were used to obtain the related genes of PD and AHS respectively, and the interaction analysis of Venn with potential active components was carried out to obtain the common target of DCHD in the treatment of the two diseases.Using STRING 11.0 and Cytoscape3.72 to analyze protein-protein interaction of common targets and screen key common targets. BioGPS was used to obtain the distribution information in organs and tissues, and the relationship between the molecules and the key functional molecules were described. Bioconductor $(R)$ was used to analyze the gene ontology (go) enrichment and the pathway analysis of the Kyoto Encyclopedia of genes and genomes (KEGG), so as to systematically predict the mechanism of "Common treatment for different diseases" of DCHD for PD and AHS.

Results: with $\mathrm{OB} \geq 30 \%$ and $\mathrm{DL} \geq 0.18$ as the screening criteria, 133 active compounds were screened out and 1034 drug targets were obtained; There are 3878 PD gene targets, 2674 AHS gene targets, 129 drug disease common targets, and 10 key targets whose median value is greater than 18;The key

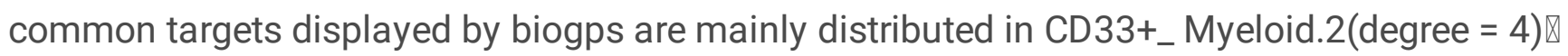

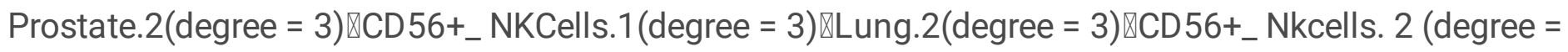
2);2281 biological processes, 65 cell components and 142 molecular functions were obtained by GO functional enrichment analysis;161 signal pathways were obtained by KEGG enrichment analysis, and the ones with higher proportion were AGE-RAGE signaling pathway in diabetic complications,PI3K-Akt signaling pathway,TNF signaling pathway,IL-17 signaling pathway,MAPK signaling pathway,HIF-1 signaling pathway,Relaxin signaling pathwa,C-type lectin receptor signaling pathway,which is mainly related to oxidative stress, glycolipid metabolism, immune inflammatory response, and neuroendocrine.

Conclusion: DCHD can achieve the effect of "Common treatment for different diseases" by acting on the common receptor of PD and AHS through multi-component, multi-target and multi-channel, providing reference for further experimental verification, potential pharmacological mechanism and clinical application.

\section{Background}


With the rapid development of social economy, people's work, life style and diet structure are also changing, which leads to the increasing prevalence of metabolic diseases and cardiovascular and cerebrovascular diseases in China. Among them, diabetes and stroke are seriously harmful to people's health and reduce the quality of life. ${ }^{[1,2]}$ PD are metabolic states with impaired fasting blood glucose, impaired glucose tolerance and / or elevated glycosylated hemoglobin $(5.7 \%-6.4 \%) .{ }^{[3]} \mathrm{A}$ number of studies have shown that PD have a causal relationship with cardiovascular disease and all-cause mortality. ${ }^{[4-8]}$ By the middle of this century, the number of PD is expected to increase by about one third. $[9,10]$ Cerebral stroke, also known as "cerebrovascular accident", is an acute cerebrovascular disease, including ischemic and hemorrhagic stroke. AHS is closely related to hyperlipidemia, diabetes, hypertension, aging of blood vessels, smoking and so on. It is a major disease endangering human health. It has the characteristics of high incidence rate, high recurrence rate, high disability rate and high mortality. ${ }^{[11]}$ Current research shows that diabetes can promote the occurrence and development of stroke ${ }^{[12]}$,And studies have shown that PD or diabetes are more common in patients with AHS. ${ }^{[13]}$ In terms of the treatment of these two diseases, more and more studies have confirmed that the pathway of brain gut axis in vivo may be used to treat metabolic diseases such as PD and other metabolic diseases at the same time to treat acute hemorrhagic cerebral apoplexy and moderate brain diseases, so as to achieve the therapeutic effect of "Common treatment for different diseases". ${ }^{[14-17]}$ The classic works of traditional Chinese medicine "Huangdi Neijing" said that "the lung and the large intestine are inside", "the heart and the small intestine are inside" and "the heart controls the mind, which is related to people's thinking and consciousness activities". It also shows that there is a treatment path of brain gut axis. ${ }^{[18-20]}$

DCHD is from treatise on Febrile Diseases written by Zhang Zhongjing. It is composed of eight kinds of herbs, namely, Chaihu(Radix Bupleuri), Huangqin(Scutellariae Radix), Dahuang (Radix Rhei Et Rhizome), Baishao(Paeoniae Radix Alba), Banxia(Arum Ternatum Thunb), Zhishi(Aurantii Fructus Immaturus), Shengjiang(Zingiber Officinale Roscoe) and Dazao(Jujubae Fructus). ${ }^{[21]}$ In the prescription, bupleurum, Scutellaria and heat clearing are used to eliminate the evil of Shaoyang; rhubarb and Fructus aurantii are used to reduce Qi, eliminate phlegm, eliminate accumulation, and reduce Yang and clear heat; peony and Fructus aurantii are used to soothe liver, relieve pain, and rhubarb are used to treat abdominal pain, while the combination of peony and Fructus aurantii can regulate qi and blood, remove heart and stomach pain; semi summer dampness and stomach depression are combined with ginger to treat nausea, but also to protect spleen and stomach; semi summer Xin When rhubarb and Scutellaria baicalensis Georgi, rhubarb and Fructus aurantii are combined with Kaiyu and Qingre, it can not only separate heat and break the knot in the Qi, remove the heat in the Qi, make the Qi mechanism unobstructed, but also separate heat and blood stasis in the blood, and regulate both qi and blood. It can be seen from the above drug composition that DCHD has the functions of activating liver depression, clearing stomach heat, regulating qi mechanism and activating blood vessels. ${ }^{[22]}$ It is commonly used in the treatment of acute pancreatitis, acute cholecystitis, diabetes, stroke, gastric and duodenal ulcer. ${ }^{[23]}$ The results showed that saikosaponin, an active component of Bupleurum chinense, has the functions of anti-tumor, anti oxidative damage, anti fibrosis and immune regulation ${ }^{[24]}$;Rhubarb, the main chemical component of 
rhubarb, can reduce the permeability of pancreatic cells by promoting the expression of tight junction protein-5 and closure protein ${ }^{[25] ;}$;Scutellarin and Baicalin in Scutellaria baicalensis Georgi can clear many kinds of free radicals, inhibit the metabolism of xanthine oxidase to produce oxygen free radicals, and be used to treat diseases related to free radicals and oxidative stress. They are effective antioxidants in Scutellaria baicalensis Georgi;, [26]Paeony can inhibit platelet aggregation and compatibility of Bupleurum can reduce blood viscosity ${ }^{[27]}$,Fructus aurantii Immaturus can promote the decomposition of fat, reduce blood lipid, and has the function of antioxidation and scavenging free radicals ${ }^{[28]}$. At the same time, by regulating the inflammatory reaction, DCHD can significantly reduce the levels of TNF - a, IL-6 and IL-8 in patients' serum. Meanwhile, regulating the oxidative reaction can increase the activity of SOD and CAT, and reduce the content of $\mathrm{MDA}^{[29]} \llbracket$ And enhance the immune response,So as to achieve the purpose of regulating $\mathrm{PD}$ and preventing and treating AHS.

Although the clinical effect of DCHD is accurate at present, for PD and AHS, "Common treatment for different diseasesr" may be achieved through "brain gut axis". However, due to the characteristics of "multi-component, multi-target, multi-channel" of traditional Chinese medicine compound, the specific mechanism of action is still unclear. It is difficult to systematically and completely elucidate its mechanism of action only by traditional pharmacological research methods, Network pharmacology integrates the ideas of system biology and multi-directional pharmacology. By building the interaction relationship between "drugs-target-disease", it combines multi-disciplinary and multi-group databases to apply to the screening of material basis, the identification of new drug targets and the study of action mechanism. The research strategy of network pharmacology integrity is consistent with the concept of

TCM integrity. ${ }^{[30-31]}$ In this study, the network pharmacology method was used to systematically explore the mechanism of DCHD in the treatment of PD and AHS from the perspective of "Common treatment for different diseases", so as to provide a reference for its in-depth study and clinical application. The flowchart of the experimental procedures of our study is shown in Fig. 1.

\section{Materials And Methods}

\subsection{Data Preparation}

\subsubsection{Collection and Treatment of Active Compounds and Targets in DCHD}

The chemical components of Chaihu(Radix Bupleuri), Huangqin(Scutellariae Radix), Dahuang (Radix Rhei Et Rhizome), Baishao(Paeoniae Radix Alba), Banxia(Arum Ternatum Thunb), Zhishi(Aurantii Fructus Immaturus), Shengjiang(Zingiber Officinale Roscoe) and Dazao(Jujubae Fructus) in DCHD were collected from the Traditional Chinese Medicine Systems Pharmacology Database and Analysis Platform (TCMSP; http://Isp.nwu.edu.cn/tcmsp.php). TCMSP is pharmacology platform for Chinese herbal medicines. In the TCMSP database, the key parameters of screening were the Oral bioavailability (OB) and Drug likeness (DL), and then to screening of the active components of Chaihu(Radix Bupleuri), Huangqin(Scutellariae Radix), Dahuang(Radix Rhei Et Rhizome), Baishao(Paeoniae Radix Alba), Banxia(Arum Ternatum Thunb), Zhishi(Aurantii Fructus Immaturus), Shengjiang(Zingiber Officinale Roscoe) and Dazao(Jujubae Fructus). 
In our study, $(\mathrm{OB}) \geq 30 \%$ and drug likeness $(\mathrm{DL}) \geq 0.18$ were selected as candidate active components using the TCMSP database, and their potential targets were retrieved.

\subsubsection{Identification of gene names.}

The screened effective targets (the duplicates were removed) were combined with similar terms to obtain the potential effective targets of all herbs in DCHD. Next, we used the UniProtKB search function in the UniProt database (UniProt; http://www.uniprot.org/) to obtain the official symbol for each protein by inputting the protein names with the species limited to "Homo sapiens". Then, we can get the right genetic symbols.

\subsubsection{Retrieval of Relevant Biological Targets for PD and AHS}

In this study, we collected Biological Targets for disease from four databases:GeneCardsv4.14

(GeneCards; http://www.genecards.org/,2020.06.10), Online Mendelian Inheritance in Man (OMIM) (OMIM; http://www.omim.org/,2020.06.10). Therapeutic Target Database (TTD). (TTD; http://www.http://db.idrblab.net/ttd/,2020.06.10) , Comparative Toxicogenomics Database (CTD) (CTD; http://www.http://ctdbase.org/,2020.06.10). The key words " Prediabetes " and " Acute hemorrhagic stroke" was input to obtain the genes related to disease. Then, we remove duplicate genes and false positive genes from four databases.

\subsection{Network construction}

To visualize the complex interactions between components and potential targets, we established the complex networks by using Cytoscape (Cytoscape; http://cytoscape.org/, ver.3.7.2). In this study, network pharmacology was used to explore the interrelationships of the herbs, their ingredients, and targets with PD and AHS, which were represented by nodes and edges. The network construction was performed as follows:(1) Venn diagram of drug targets with the genes associated with a disease;(2) the active compounds-active compounds target network of DCHD was built; (3) the herb-compound target- disease target network was built via linking the eight DCHD herbs with compound targets of each herb, and disease targets.

We constructed the networks by utilizing the network visualization software Cytoscape. In this network, there are three important indices including degree, node betweenness, and closeness to evaluate every node. At first, Degree indicates the number of edges between a node and other nodes in a network. ${ }^{[32]}$ Secoundly, Node betweenness evaluates the participation of a node in the shortest parts of the network and reflects the ability of node stop proceed the rate of information flow in the network as well. ${ }^{[33]}$ At last, Closeness refers to the inverse of the sum of the distance from a node to other nodes. This indicates determine whether a target protein important basis for the key targets. ${ }^{[34]}$

\subsection{Protein-Protein Interaction Data}


The PPI network was constructed using the STRING platform(https://string-db.org/), and the species was set as "Homo sapiens". The minimum threshold of interaction was set as "high confidence data $>0.7$ ". Meanwhile, the PPI network was constructed via utilizing the network visualization software Cytoscape3.7.2. It is important to explore protein interactions and their interaction networks to understand cellular organization, bioprocesses, and functions.

\subsection{Enrichment of Gene Ontology (GO)Pathway and the Kyoto Encyclopedia of Genes and Genomes (KEGG) Pathway}

GO is an international standard classification system for gene function. GO enrichment analyses can reveal the functional changes in these targets in three respects: molecular biological function, biological process, and cellular components. And then KEGG metabolic pathway enrichment analysis was carried out to study the main metabolic pathway of YQQDC in the treatment of COVID-19. We used Bioconductor (R)v3.6.2 (Bioconductor (R); http://bioconductor.org/)for analyses. The statistics were collected by the ClueGO and CluePedia plugins with the FDR set as $\leq 0.05$.

\section{Results}

\subsection{Identification of the active compounds in DCHD}

DCHD consists of Chaihu(Radix Bupleuri), Huangqin(Scutellariae Radix), Dahuang (Radix Rhei Et Rhizome), Baishao(Paeoniae Radix Alba), Banxia(Arum Ternatum Thunb), Zhishi(Aurantii Fructus Immaturus), Shengjiang(Zingiber Officinale Roscoe) and Dazao(Jujubae Fructus). Using the TCMSP database, the main active components of each herbal medicine should meet the requirements of $\mathrm{OB}$ value $\geq 30 \%$ and $D L$ value $\geq 0.18$. 140 active compounds were retrieved, including 18 in $\mathrm{CH}, 36$ in $\mathrm{HQ}, 15$ in DH, 13 in BS, 13 in BX, 22 in ZS, 5 in SJ, 29 in DZ. A total of 133 highly active compounds were obtained by eliminating 18 repetitive compounds, which are listed in Table 1.

\subsection{Identification of targets of DCHD, PD and AHS}

We screened out 1034 effective targets of 80 of 133 high active compounds, including 276 in $\mathrm{CH}, 242$ in HQ, 48 in DH, 61 in BS, 73 in BX, 117 in ZS, 32 in SJ, and 185 in DZ. (Tables S1-S8). Simultaneously, We screened 3878 important gene targets related to PD and 2674 important gene targets related to AHS (Table S9-S10). In other words, 129 genetic symbols may be the key to DCHD 's treatment of PD and AHS.

\subsubsection{Drug Target - Disease Target Analysis}

We through the analysis of Venn's diagram can get the conclusion that there are 129 overlaps between 3878 PD related gene targets, 2674 AHS related gene targets and 1034 effective disease targets.(Figure2)

\subsubsection{Drug Ingredients-Target Network Analysis}


The drug composition target network consists of 293 nodes (80 compounds and 206 compound targets in DCFT) and 785 edges.As shown in Figure 3, A1 (kaempferol) is $\mathrm{CH}$, and BS has common components; $\mathrm{A} 2$ (quercetin) is a common component of $\mathrm{CH}$ and $\mathrm{DZ} ; \mathrm{A} 3$ (stigmasterol) is a common component of HQ, BX, SJ, DZ;A4 (baicalein) is a common component of $\mathrm{HQ}, \mathrm{BX}$;A5 (eriodyctiol (flavanone)) is $\mathrm{HQ}$ and ZS;A6 (beta sitosterol) is composed of $\mathrm{DH}, \mathrm{BS}, \mathrm{BX}$ and $\mathrm{SJ} ; \mathrm{A7}$ (sitosterol) is $\mathrm{HQ}$ and BS;A8 ((-) - catechin) is the common component of DH, DZ;A9 (mairin,(+) - catechin) which is a common component of BS and DZ; The network shows that many compound targets can be adjusted by a variety of compounds. In addition, we can also roughly observe the relationship between the active compounds and their targets.

\subsubsection{Herb Compounds - disease Target- disease Network Analysis}

This network was built to show the relationship between eight herbs, compound, PD and AHS targets, PD and AHS(Figure 4). Analysis of this network revealed that the network map contained 201 nodes and 898 edges. The 201 nodes included 69 active components of DCHD, 8 herbs, 2 disease and 129 targets of PD and AHS targets. Figure 3 shows that DCHD may affect drug targets by controlling related proteins (compound targets).

\subsection{Screening and analysis of key shared targets}

\subsubsection{Analyses of a PPI Network}

Through the results of Venn graph, we get 129 repeat important targets. In order to illuminate the significance of degree in compound targets, we created a PPI network about the relationship of the common targets between compounds and PD and AHS. (Figure 5) This PPI network consisting of 129 nodes and 492 edges. Meanwhile, the PPI network was constructed via utilizing the network visualization software Cytoscape3.7.2. (Figure 5) In this network, the node size and color are used to reflect the number of combined targets (degree). As shown in Figure 6, the darker color and the larger circle indicates a higher degree. What's more, the bar plot of the PPI network analysis results showed that AKT1(degree=31), JUN(degree=29), RELA(degree=28), IL6(degree=26), MAPK1(degree=26), APP(degree=20), MAPK14 (degree=20), EGFR(degree=19), MAPK8(degree=19),VEGFA(degree=19) were the pivotal targets in this network. (Figure 7) In this figure, the $x$-axis represents the number of neighboring proteins of the target protein. The $y$-axis represents the target protein. From the above figures, we indicating that there were 10 core targets obtained after PPI network analysis, as well as the critical role in the treatment of PD and AHS.

\subsubsection{Network construction and topological analysis of "key target organ tissue"}

Key shared targets AKT1, Jun, rela, IL6, mapk1, app, Mapk14, EGFR, Mapk8, VEGFA were imported into biogps to obtain the distribution in organs and tissues, and relevant information was imported into Cytoscape 3.72 to build a "key target organ tissue" network map and carry out topological analysis(Figure 8) . In the figure, the blue diamond represents the disease, and the pink triangle node is the key target, The 
red circle node is the organ tissue, and the circle size represents the size of the degree value. According to the degree value, the information of the first five distribution organs is as follows: CD33+_

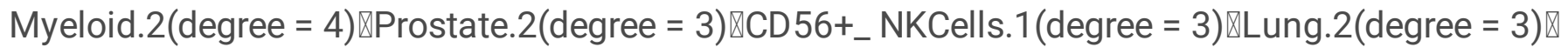
CD56+_NKCells.2 (degree $=2$ ) $\llbracket$ suggesting that the above organs and tissues play an important role in the treatment of PD and AHS.

\subsection{Analyses of Enrichment of GO Pathways}

Using the GO enrichment analysis function of the Bioconductor (R), We carried out GO enrichment analyses to further determine the functions of these shared targets from three aspects, and $G O$ entries were determined using a false discovery rate (FDR) of $<0.05$. Through the analysis of GOBP, GOCC, GOMF, we obtained 2281 biological processes, 65 cell components and 142 molecular functions. As shown in Figure 9(a,b,c), Top 20 functional terms were enriched in the biological process category, such as response to lipopolysaccharide,response to oxidative stress,response to nutrient levels,reactive oxygen species metabolic process,response to steroid hormone,regulation of apoptotic signaling pathway and muscle cell proliferation.Top 20 functional terms were enriched in the cellular components'category, such as membrane raft,cytoplasmic vesicle lumen,secretory granule lumen, mitochondrial outer membrane,nuclear chromatin,platelet alpha granule, endoplasmic reticulum lumen,protein kinase complex,RNA polymerase II, transcription factor complex and serine/threonine protein kinase complex. Additionally, Top 20 functional terms were enriched in the molecular function category, such as cytokine activity,heme binding,cytokine receptor bindingtetrapyrrole binding,nuclear receptor activity,transcription factor activity, and direct ligand regulated sequence-specific DNA binding. Undoubtedly, these biological processes were all involved in the pathogenesis of PD and AHS, so they may serve as a potential therapeutic mechanism for PD and AHS.

\subsection{Analyses of Enrichment of KEGG Pathways}

Analyses of enrichment of the KEGG pathway were also carried out using Bioconductor (R) and ClueGO. $(p<0.01)$ The pathways of 129 proteins involved in PPI network were analyzed via pathway enrichment, and 161 signaling pathways were obtained. Figure 10 shows the top 20 pathways for DCHD in the treatment of PD and AHS target. The functionally grouped network of enriched categories was generated for the target genes using ClueGO and CluePedia (Figure 11). In addition, we established a drug-targetpath network \which clearly indicates that DCHD may achieve its purpose of treating PD and AHS through multiple targets and pathways. Figure 13 shows the proportion of each group associated with 129 targets. The most significant KEGG terms of the target genes included Fluid shear stress and atherosclerosis Kaposi sarcoma-associated herpesvirus infection,AGE-RAGE signaling pathway in diabetic complications,Human cytomegalovirus infection,Hepatitis B,PI3K-Akt signaling pathway,TNF signaling pathway,Hepatitis C Prostate cancer,Influenza A,Epstein-Barr virus infection,Human papillomavirus infection,IL-17 signaling pathway, Proteoglycans in cancer囚MAPK signaling pathway,MicroRNAs in cancer, Measles, Human immunodeficiency virus 1 infection,Salmonella infection,HIF-1 signaling pathway. Figure 13 shows the AGE-RAGE signaling pathway in diabetic 
complications ,PI3K-Akt signaling pathway,IL-17 signaling pathway and MAPK signaling pathway were discussed to illustrate the underlying therapeutic mechanisms of DCHD for MAPK signaling pathway treatment.

\section{Discussion}

The composition of traditional Chinese medicine compound is complex, in which the quantity and dosage of traditional Chinese medicine are slightly increased or decreased, which can achieve different clinical effects, which is very delicate.Many traditional Chinese medicine compounds can achieve the effect of "Common treatment for different diseases", but the pharmacophore and mechanism of action need to be studied.

The classic prescription DCHD, originated from the treatise on febrile diseases, has the effect of reconciling Shaoyang, clearing away Yang and clearing away Yang Ming. It is widely used in clinical practice. ${ }^{[35]}$ Modern pharmacology has proved that Saikosaponin-d in Bupleurum can not only effectively inhibit a variety of inflammatory reactions, but also inhibit the elevation of blood lipid level; Scutellaria has the effects of cholagogic, liver protecting and blood lipid reducing, and baicalin and baicalin can interfere with arachidonic acid metabolic pathway and inhibit the activity of cytokines, produce antipyretic and anti-inflammatory effects; rhubarb has the effects of reducing serum cholesterol, liver protecting and blood pressure reducing; Paeony has the functions of protecting hepatocytes, promoting hepatocyte regeneration, reducing lipid and inhibiting hepatocyte fibrosis. ${ }^{[36]}$ At present, DCHD is mainly used in the disease of "Shaoyang Yangming combined disease". ${ }^{[35]}$ Through clinical practice, it is found that $\mathrm{DCHD}$ is not only widely used in diabetes, hepatobiliary disease, but also has certain curative effect in the treatment of acute hemorrhagic stroke related diseases. ${ }^{[37]}$ Traditional Chinese medicine is guided by the principle of holistic view and syndrome differentiation and treatment, Network pharmacology is based on the theory of system biology, through the construction of interaction network among drugs, diseases and targets, and then systematically and integrally study the action mechanism of drugs with multiple targets and channels. They have common points in philosophical thinking. ${ }^{\left[{ }^{[6-38]}\right.}$ Therefore, this paper uses the method of network pharmacology to explore the "Common treatment for different diseases" PD and AHS of DCHD Mechanism of action.

In this study, through network analysis, we found that DCHD has 129 common targets in the treatment of PD and AHS, and further extracted 10 key common targets by protein interaction network analysis. According to the topological index degree in network analysis, AKT1, JUN, RELA, IL6, MAPK1, APP, MAPK14, EGFR,MAPK8, VEGFA, which are closely related to oxidative stress, glycolipid metabolism, immune inflammatory response, and neuroendocrine regulation are the key common targets, AKT1 (aktserine / threoninekinase1) is one of the three serine / threonine protein kinases (AKT1, AKT2, Akt3) [39]. It regulates the biological processes by participating in multiple signaling pathways related to inflammation, immunity, metabolism and cell proliferation, such as AGE-RAGE, FOXO, EGFR tyrosine kinase inhibitor resistance, PI3K Akt and MAPK. Interleukin (IL) is one of the key common targets, 
including IL2, IL6, IL $10^{[40]}$. IL6 is produced by monocyte macrophages, Th2 cells (thelper2cell), vascular endothelial cells, etc. nitric oxide synthetase 2 (NOS2) can promote IL6 synthesis and participate in inflammatory response through the production of nitric oxide as a kind of messenger molecule In addition, IL-2 is produced by T cells, IL-10 is produced by Th2 cells and monocyte macrophages, all of which are involved in immune response by activating $T$ cells, promoting $B$ cell proliferation and secreting antibodies, activating macrophages, etc The regulation of disease and inflammation. Mapk1, mapk3 and Mapk14 in mitogen activated protein kinase (MAPK) family are another kind of key common targets ${ }^{[41]}$. MAPK family widely exists in various mammalian tissues. MAPK family can be activated by extracellular stimulation, such as cytokines, neurotransmitters, hormones, cell stress and cell adhesion. Through threestage kinase mode, MAPK family can jointly regulate anti-inflammatory effect There are many important physiological and pathological processes such as cell growth, differentiation, stress adaptation and so on ${ }^{[42]}$.Epidermal growth factor receptor (EGFR) binds to ligands and activates multiple signal cascades to convert extracellular signals into appropriate cellular responses to activate major downstream signaling pathways such as rasraf-mek-erk, PI3K-Akt ${ }^{[43]}$.Vascular endothelial growth factor A (VEGFA) promotes blood flow and glucose metabolism by promoting endothelial cell proliferation, migration and inhibiting apoptosis ${ }^{[44]}$.Tumor necrosis factor (TNF) can not only regulate immune function, but also affect blood vessels, blood flow and blood glucose ${ }^{[45]}$.

Through the go enrichment analysis function of biological conductor $(\mathrm{R})$, we found that DCHD plays an important role in the treatment of PD and AHS, including the response to lipopolysaccharide, the response to oxidative stress, the response to nutritional level, the process of active oxygen metabolism, the response to steroid hormones, regulation and diffusion of apoptosis signaling pathway and muscle cells, etc. The molecular functions play an important role, such as cytokine activity, heme binding, cytokine receptor binding tetrapyrrole binding, nuclear receptor activity, transcription factor activity and direct ligand regulated sequence specific DNA binding, MAP kinase activity and so on, which are related to the predicted signal pathway.

In order to further explore the common key signal pathways, we used the biological conductor $(R)$ and cluego to enrich the KEGG signal pathway, and found that the higher signal pathways were AGE-RAGE signaling pathway indiabetic complications, PI3K aktsignaling pathway, TNF signaling pathway, IL-17 signaling pathway, MAPK signaling pathway, HIF-1 signaling pathway, Relaxin signaling pathway, C-type lectin receptor signaling pathway, etc., are mainly related to oxidative stress, metabolism, immune inflammation and neuroendocrine. AGE-RAGE signaling pathway can not only cause glycolipid metabolism disorder and oxidative stress enhancement, but also induce MAPK, JAK-STAT and PI3K Akt signaling pathway to promote inflammation and promote the development of diabetes ${ }^{[46]}$,Previous studies also found that regulating AGE-RAGE signaling pathway can alleviate oxidative stress in prefrontal cortex and hippocampus of depressed rats and improve stroke symptoms ${ }^{[47]}$, It has been reported that DCHD can reduce the content of oxygen free radicals (such as no, Co, MDA) and improve the level of SOD ${ }^{[48]}$.It is also confirmed that Th17 and IL-17 signaling pathway affect diabetes mellitus and acute hemorrhagic stroke by regulating immune inflammatory response. ${ }^{[49-52]}$ It is also proved that 
DCHD can reduce the levels of hs CRP, IL- 6 and TNF - $a$ in serum, and reduce the damage of inflammatory factors to the focus and surrounding cells. ${ }^{[53,54]}$

The common signaling pathways regulating immune and inflammatory responses in PD and AHS also include EGFR tyrosine kinase inhibitor resistance, TNF, PI3K Akt, toll like receptor, MAPK, JAK stat,

cytokine cytokine receptor interaction and other signaling pathways ${ }^{[55-60]}$. HIF-1 signaling pathway has cell-specific response to vascular endothelial cells, smooth muscle cells and macrophages. By up regulating VEGF, NO, ROS and PDGF, it can cause endothelial cell dysfunction, angiogenesis and inflammatory response, and affect glucose metabolism and blood oxygen content, ${ }^{[61]}$ On the other hand, DCHD can reduce blood viscosity and fibrinogen level, inhibit platelet aggregation, promote thrombolysis, correct abnormal Hemorheology and increase effective circulation perfusion. The results are confirmed by each other. ${ }^{[62]}$

In order to further predict the possible organs / tissues of DCHD, it was found that the key common targets were mainly distributed in CD33 through the analysis of biogps+_ Myeloid.2 (degree $=4) \square$

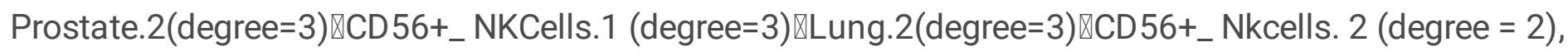
which is closely related to the metabolism of glycolipid energy, the proliferation and migration of vascular smooth muscle cells, the active oxygen metabolism process involved in acute hemorrhagic stroke, the response to steroid hormones, the regulation of apoptosis signal pathway and muscle cells, in a certain extent reflects the reliability of the network construction method The following experimental verification provides the basis.

\section{Conclusions}

To sum up, through the prediction of network pharmacology, it is found that DCHD may through the common signal pathway to regulate oxidative stress, glycolipid metabolism, immune inflammatory response and neuroendocrine regulation to achieve the "Common treatment for different diseases" for PD and AHS, which is consistent with the current research on the mechanism of PD and AHS to some extent, it indicates the reliability and accuracy of network pharmacology prediction results, but further experimental verification is needed to clarify the influence of DCHD on the above prediction key targets and signal pathways. This study shows that DCHD has the characteristics of multi-component, multitarget and multi-channel, which can provide a reference for the next stage to study the mechanism of DCHD, and also provide a basis for the rational clinical application and new drug development.

\section{Declarations}

Acknowledgements Not applicable.

\section{Authors' contributions}

Xiaolin Zhang, Mingjun Liu and Di Cao conceived and designed the research methods. 
Di Cao and Dehui Ma collected the data.

Xiaolin Zhang, Qi Zhang date analysis, Visualization.

Xiaolin Zhang and Di Cao wrote the paper.

Mingjun Liu Resources, Supervision,

All authors read and approved the final manuscript.

Funding This work was supported by the School construction project of State Administration of traditional Chinese medicine (Grant Number LPGZS22014-11) and The Jilin Local Standard Construction Project (Grant Number: DBXM097-2020).

Availability of data and materials The data and materials generated or analyzed during this study are available from the corresponding author on reasonable request.

Consent for publication The manuscript is approved by all authors for publication.

Competing interests The authors declare that they have no competing interests.

\section{Abbreviations}

$\mathrm{DCHD}=$ Dachaihu Decoction;PD=Prediabetes;AHS=Acute hemorrhagic stroke(AHS);CH=Chaihu(Radix Bupleuri); $\mathrm{HQ}=$ Huangqin(Scutellariae Radix); $\mathrm{DH}=$ Dahuang (Radix Rhei Et Rhizome);BS=Baishao(Paeoniae Radix Alba);BX=Banxia(Arum Ternatum Thunb);ZS=Zhishi(Aurantii Fructus Immaturus);SJ=Shengjiang(Zingiber Officinale Roscoe);DZ=Dazao(Jujubae Fructus)

\section{References}

1. MD C, T S, PZ Z, KR T, B O, DR O, JE S, RJ T: Benefit of lifestyle-based T2DM prevention is influenced by prediabetes phenotype. Nature reviews Endocrinology 2020.

2. AN S, M J, HS O, J R, G B, S D, AM S: Biomarker Application for Precision Medicine in Stroke. Translational stroke research 2019.

3. American Diabetes Association. Classification and Diagnosis of Diabetes. Diabetes Care. 2017 Jan;40(Suppl 1):S11-S24.

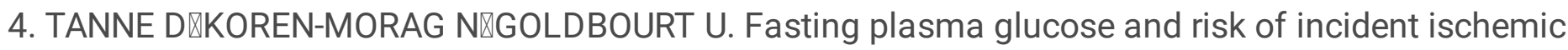
stroke or transient ischemic attacks $₫ a$ prospective cohort study. Stroke $2004 \rrbracket 35 \rrbracket 10 \rrbracket \rrbracket 2351-2355$.

5. BLAKE D R囚MEIGS J B囚MULLER D C『et al. Impaired glucose tolerance囚but not impaired fasting

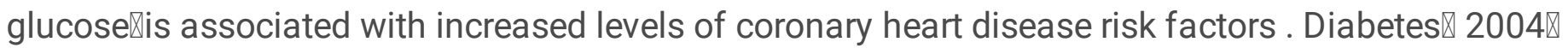
53囚8ख $2095-2100$. 
6. SINGLETON J R囚SMITH A G囚RUSSELL J W囚et al. Microvascular complications of impaired glucose tolerance . Diabetes $₫ 2003 \llbracket 52 \rrbracket 12 \otimes \otimes 2867-2873$.

7. FORD E S\&ZHAO G囚LI C. Pre-diabetes and the risk for cardiovascular disease『a systematic review of the evidence. J Am Coll Cardiol $₫ 2010 \otimes 55 \rrbracket 13 \otimes \bigotimes 1310-1317$.

8. Huang $Y$, Cai X, Mai W, Li M, Hu Y . Association between prediabetes and risk of cardiovascular disease and all cause mortality: systematic review and meta-analysis. BMJ. 2016 Nov 23;355:i5953.

9. Danaei G, Finucane MM, Lu Y, et al. National, regional, and global trends in fasting plasma glucose and diabetes prevalence since 1980: systematic analysis of health examination surveys and epidemiological studies with 370 country-years and 2.7 million participants. Lancet. 2011;378(9785):31-40.

10. International Diabetes Federation. Chapter 3 - Global picture: Diabetes as a growing problem. IDF Diabetes Atlas, Eighth Edition, 2017.

11. Broke JG, Lankhorst JG, Rumping K, et al. The long term out-come of arm function after stroke: results of a follow-up study. Disabil Rehabil, 1999, 21(8):357-364.

12. Cui Yanrong, Zhou Qi, Zhu Xiangdong. Effect of Dachaihu Decoction on oxidative stress-induced pancreatic $\beta$ cell damage in type 2 diabetic rats [J / OL]. World science and technology modernization of traditional Chinese medicine: 1-6 [2020-06-19].

13. Xue libeng, Wang Xiao, Shi Qianqian, Li Qin, Liu Yong, an Zixiong. Study on the effect of Dachaihu Decoction on the short-term prognosis of stroke patients with phlegm heat and viscera excess type [J]. Journal of modern integrated Chinese and Western medicine, 2020,29 (14): 1513-1516 + 1521.

14. $B T, L P, A S, D B, L B, P R, V$ G: Gut-Brain Axis and Neurodegeneration: State-of-the-Art of Meta-Omics Sciences for Microbiota Characterization. International journal of molecular sciences 2020, 21(11).

15. C P, L A, V C, R C, M F, C B: Microbiota-gut-brain axis in health and disease: Is NLRP3 inflammasome at the crossroads of microbiota-gut-brain communications? Progress in neurobiology 2020 , 191:101806.

16. M O, H J, T T, A M, L S, G P, RA B: The Role of the Microbiota-Gut-Brain Axis and Antibiotics in ALS and Neurodegenerative Diseases. Microorganisms 2020, 8(5).

17. R Y, CT H, X Z: Interaction Between Tea Polyphenols and Intestinal Microbiota in Host Metabolic Diseases from the Perspective of the Gut-Brain Axis. Molecular nutrition \& food research 2020:e2000187.

18. Li Sicheng, Wang Hua, Wu Song, Wu fan, Duan can. Application of the theory of "heart and small intestine" in myocardial ischemia disease. Shi Zhen Guo Yi Guo Yao, 2020,31 (01): 153-155.

19. He Guliang, Wu Tao, Lu Fangguo. The clinical application and experimental research progress of "lung and large intestine" theory . Hunan Journal of traditional Chinese medicine, 2018,34 (12): 150152.

20. Guo Zongyao. Based on the theory of "heart and small intestine outside and inside", to explore the effect of Yangxin Tongmai Recipe on intestinal flora of rats with coronary heart disease and blood stasis syndrome. Hunan University of traditional Chinese medicine, 2017. 
21. HIDEAKI K, TAKA-AKI M, YOSHIHISA N, et al. A crucial role of MafA as a novel therapeutictarget for diabetes[J]. Journal of Biological Chemistry, 2005, 280(15): 15047.

22. Wei XiuXiu, Gou Xiaowen, Zhao Linhua, Liu Sheng, Tong Xiaolin. Application of state target syndrome differentiation in obese type 2 diabetes mellitus of liver and stomach depression heat type -- Dachaihu Decoction plus Coptis, Anemarrhena and Chishao [J]. Liaoning Journal of traditional Chinese medicine, 2020,47 (03): 1-3.

23. LF D, XF X, LJ Z, F L, XQ Z, N W, JW F, JQ X, H Z: Dachaihu decoction ameliorates pancreatic fibrosis by inhibiting macrophage infiltration in chronic pancreatitis. World journal of gastroenterology 2017, 23(40):7242-7252.

24. Liu Dan, Wang Jiahe. Research progress on antitumor mechanism of saikosaponin [J]. Modern medicine and clinical, 2018, 33 (01): 203-208.

25. Wang Yijun, Feng Shuhan, Cheng Jintang, et al. Research progress of anthraquinones in rhubarb. Chinese Journal of experimental pharmacology, 2018, 24 (13): 227-234.

26. Zheng Yongfeng, Wang Jiajing, Fu Chaomei, et al. Research progress of chemical constituents and pharmacological action of Scutellaria baicalensis Georgi. Chinese patent medicine, 2016, 38 (01): 141-147.

27. Xiao Gang, sun Xinxin, Wang Shijie, et al. Study on the protective effect and mechanism of TGP on atherosclerosis in rats. Chinese Journal of evidence based cardiovascular medicine, 2017, 9 (3): 273276.

28. Xia Hanxing, Zhang Ye. Effect of Zhishi Xiebai Guizhi Decoction on blood lipid and vascular endothelial function in hyperlipidemia rats. Chinese Journal of experimental formulary, 2012, 18 (10): 224-226.

29. Ren Xueming, Zhu Yubo. Effect of Dachaihu Decoction on inflammatory response and oxidative stress of acute pancreatitis with stagnation of liver Qi. Journal of Guizhou Medical University, 2017, 42 (07): 821-824.

30. Wang Ping, Tang Shihuan, Su Jin, et al. Modern research progress of traditional Chinese medicine based on integrated pharmacology. Chinese Journal of traditional Chinese medicine, 2018, 43 (7): 1297.

31. Wang Xuan, Yu He, Bai Chen, et al. Exploring the pharmacological mechanism of biochemical Decoction in the treatment of small abdominal pain based on network pharmacology. Chinese Journal of traditional Chinese medicine, 2019, 44 (10): 2124.

32. P. V. Missiuro, K. Liu, L. Zou et al., "Information flow analysis of interactome networks," PLoS Computer Biology, vol. 5,no. 4, Article ID e1000350, 2009.

33. K. Raman, N. Damaraju, and G. K. Joshi, "The organizational structure of protein networks: revisiting the centrality lethality hypothesis,"Systems and Synthetic Biology, vol. 8, no. 1,pp. 73-81, 2014.

34. Y. Zhang, M. Bai, B. Zhang et al.,"Uncovering pharmacological mechanisms of Wu-tou decoction acting on rheumatoid arthritis through systems approaches: drug-target prediction, network analysis and experimental validation,"Science Reports, vol. 5, p. 9463, 2018. 


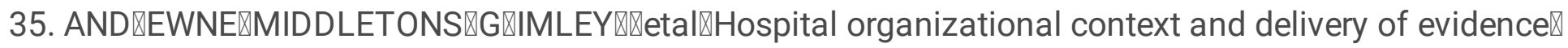
based stroke care: across $\llbracket$ sectional study.ImplementationScience,2019,14(1):6】

36. ZHANGYQ,MAOX,GUOQY,etal.NetworkpharmacologybasedapproachescaptureessenceofChineseherbalmedicines.ChinHerbMed,2016,8(2):107.

37. Yang Jingyu, Wang Rui, Jin Tao, et al. Exploring the material basis and molecular mechanism of Dachengqi Decoction in the treatment of acute pancreatitis based on network pharmacology [J]. Chinese Journal of traditional Chinese medicine, 2019, DOI: 10.19540/j.cnki.cjcmm.20191210.401.

38. Huang Meiwen, Yang Huajie, Zhou Xiaochun, et al. Application and Prospect of network pharmacology in the study of ethnic medicine Chinese Journal of traditional Chinese medicine, 2019, 44 (15): 3187.

39. HÄGGBLAD S S, MORTENSEN A C, HAGLÖF J, et al. Different functions of AKT1 and AKT2 in molecular pathways, cell migration and metabolism in colon cancer cells. Int J Oncol, 2017, 50: 5.

40. DEPALMA R G, HAYES V W, CHOW B K, et al. Ferritin levels, inflammatory biomarkers, and mortality in peripheral arterial disease: a substudy of the iron ( $\mathrm{Fe}$ ) and atherosclerosis study (FeAST) trial. J Vasc Surg, 2010, 51: 1498.

41. REUSTLE A, TORZEWSKI M. Role of p38 MAPK in atherosclerosis and aortic valve sclerosis. Int J Mol Sci, 2018,19(12):3761.

42. HASHIMOTO R, KAKIGI R, NAKAMURA K, et al. LPS enhances expression of CD204 through the MAPK/ERK pathway in murine bone marrow macrophages. Atherosclerosis, 2017, 266: 167.

43. ZEBOUDJ L, GIRAUD A, GUYONNET L, et al. Selective EGFR (epidermal growth factor receptor) deletion in myeloid cells limits atherosclerosis-brief report[J]. Arterioscler Thromb Vasc Biol, 2018, 38: 114.

44. HU S, LIU Y F, YOU T, et al. Semaphorin 7A promotes VEGFA/VEGFR2-mediated angiogenesis and intraplaque neovascularization in mice[J]. Front Physiol, 2018, 9: 1718.

45. JEAN C, PIERRE Y, WU J H, et al. USP20 (ubiquitin-specific protease 20) inhibits TNF (tumor necrosis factor)-triggered smooth muscle cell inflammation and attenuates atherosclerosis[J]. Arterioscler Thromb Vasc Biol, 2018, 38: 2295.

46. LEE T W, KAO Y H, CHEN Y J, et al. Therapeutic potential of vitamin D in AGE/RAGE-related cardiovascular diseases. Cell Mol Life Sci, 2019, 76: 4103.

47. ERGENC M, OZACMAK H S, TURAN I, et al. Melatonin reverses depressive and anxiety like-behaviours induced by diabetes: involvement of oxidative stress, age, rage and $\mathrm{S} 100 \mathrm{~B}$ levels in the hippocampus and prefrontal cortex of rats[J]. Arch Physiol Biochem, 2019, doi: 10.1080/13813455.2019.1684954.

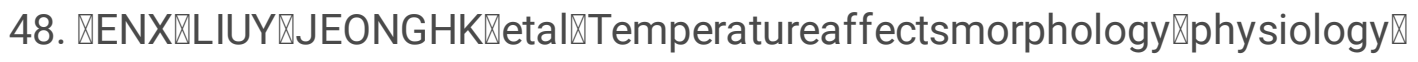
andbiochemistryofplugseedlingsofAstragalusmembranaceus $\mathbb{Z}$ ActaPhysiologiaePlantarum,2019,41(1):9囚

49. LIN J, CHANG W, DONG J C, et al. Thymic stromal lymphopoietin over-expressed in human atherosclerosis: potential role in Th17 differentiation[J]. Cell Physiol Biochem, 2013, 31: 305. 
50. ALLAM G, ABDEL M A, GABER A M. The pleiotropic role of interleukin-17 in atherosclerosis. Biomed Pharmacother, 2018, 106: 1412.

51. BEUREL E, LOWELL J A, JOPE R S. Distinct characteristics of hippocampal pathogenic T17 cells in a mouse model of depression. Brain Behav Immun, 2018, 73: 180.

52. NADEEM A, AHMAD S F, AL-HARBI N O, et al. IL-17A causes depression-like symptoms via NFKB and p38MAPK signaling pathways in mice: Implications for psoriasis associated depression. Cytokine, 2017, 97: 14.

53. Shi Jingwei, Liu min. based on the therapeutic effect of Dachaihu Decoction on acute stroke and the significance of syndrome differentiation of six meridians [ $\mathrm{J}]$. Chinese Journal of traditional Chinese medicine, 2020,38 (05): 155-157.

54. Yang Aoran, Tian Xin, Wu Qiong. Clinical observation of Dachaihu Decoction on acute cerebral infarction [J]. Journal of Hubei University of traditional Chinese medicine, 2015, 17 (1): 37-39.

55. WANG L T, HUANG Z Q, HUANG W J, et al. Inhibition of epidermal growth factor receptor attenuates atherosclerosis via decreasing inflammation and oxidative stress[J]. Sci Rep, 2017, 8: 45917.

56. PIRL W F, SOLIS J, GREER J, et al. Epidermal growth factor receptor tyrosine kinase inhibitors and depression[J]. J Clin Oncol, 2009, 27: e49.

57. KONG X L, ZHANG Z Y, FU T, et al. TNF-a regulates microglial activation via the NF-kB signaling pathway in systemic lupus erythematosus with depression[J]. Int J Biol Macromol, 2019, 125: 892.

58. MENG L B, SHAN M J, QIU Y, et al. TPM2 as a potential predictive biomarker for atherosclerosis[J]. Aging, 2019, 11: 6960.

59. MA K, ZHANG H X, WEI G H, et al. Identification of key genes, pathways, and miRNA/mRNA regulatory networks of CUMS-induced depression in nucleus accumbens by integrated bioinformatics analysis[J]. Neuropsychiatr Dis Treat, 2019, 15: 685.

60. HAN X B, KOU J Y, ZHENG Y H, et al. ROS generated by upconversion nanoparticle-mediated photodynamic therapy induces autophagy via PI3K/AKT/mTOR signaling pathway in M1 peritoneal macrophage[J]. Cell Physiol Biochem, 2019, 52: 1325.

61. JAIN T, NIKOLOPOULOU E A, XU Q B, et al. Hypoxia inducible factor as a therapeutic target for atherosclerosis[J]. Pharmacol Ther, 2018, 183: 22.

62. LI G F, ZHAO M, CHENG X, et al. FG-4592 improves depressive-like behaviors through HIF-1-mediated neurogenesis and synapse plasticity in rats[J]. Neurotherapeutics, 2019, doi: 10.1007/s13311-01900807-3.

\section{Tables}


Table 1 The list of 65 compounds of Radix Salviae and their OB and DL

\begin{tabular}{|c|c|c|c|}
\hline mol ID & Compound & $\mathrm{OB}$ & DL \\
\hline MOL001645 & Linoleyl acetate & 42.1 & 0.2 \\
\hline MOL002776 & Baicalin & 40.12 & 0.75 \\
\hline MOL000449 & Stigmasterol & 43.83 & 0.76 \\
\hline MOL000354 & isorhamnetin & 49.6 & 0.31 \\
\hline MOL000422 & kaempferol & 41.88 & 0.24 \\
\hline MOL004598 & 3,5,6,7-tetramethoxy-2-(3,4,5-trimethoxyphenyl)chromone & 31.97 & 0.59 \\
\hline MOL004609 & Areapillin & 48.96 & 0.41 \\
\hline MOL013187 & Cubebin & 57.13 & 0.64 \\
\hline MOL004624 & Longikaurin A & 47.72 & 0.53 \\
\hline MOL004628 & Octalupine & 47.82 & 0.28 \\
\hline MOL004644 & Sainfuran & 79.91 & 0.23 \\
\hline MOL004648 & Troxerutin & 31.6 & 0.28 \\
\hline MOL004653 & (+)-Anomalin & 46.06 & 0.66 \\
\hline MOL004702 & saikosaponin c_qt & 30.5 & 0.63 \\
\hline MOL004718 & a-spinasterol & 42.98 & 0.76 \\
\hline MOL000490 & petunidin & 30.05 & 0.31 \\
\hline MOL000098 & quercetin & 46.43 & 0.28 \\
\hline MOL000096 & (-)-catechin & 49.68 & 0.24 \\
\hline MOL001689 & acacetin & 34.97 & 0.24 \\
\hline MOL000173 & wogonin & 30.68 & 0.23 \\
\hline MOL000228 & (2R)-7-hydroxy-5-methoxy-2-phenylchroman-4-one & 55.23 & 0.2 \\
\hline MOL002714 & baicalein & 33.52 & 0.21 \\
\hline MOL002908 & 5,8,2'-Trihydroxy-7-methoxyflavone & 37.01 & 0.27 \\
\hline MOL002909 & 5,7,2,5-tetrahydroxy-8,6-dimethoxyflavone & 33.82 & 0.45 \\
\hline MOL002910 & Carthamidin & 41.15 & 0.24 \\
\hline MOL002911 & 2,6,2',4'-tetrahydroxy-6'-methoxychaleone & 69.04 & 0.22 \\
\hline MOL002913 & Dihydrobaicalin_qt & 40.04 & 0.21 \\
\hline
\end{tabular}




\begin{tabular}{|c|c|c|c|}
\hline MOL002914 & Eriodyctiol (flavanone) & 41.35 & 0.24 \\
\hline MOL002915 & Salvigenin & 49.07 & 0.33 \\
\hline MOL002917 & 5,2',6'-Trihydroxy-7,8-dimethoxyflavone & 45.05 & 0.33 \\
\hline MOL002925 & $5,7,2$ ',6'-Tetrahydroxyflavone & 37.01 & 0.24 \\
\hline MOL002926 & dihydrooroxylin A & 38.72 & 0.23 \\
\hline MOL002927 & Skullcapflavone II & 69.51 & 0.44 \\
\hline MOL002928 & oroxylin a & 41.37 & 0.23 \\
\hline MOL002932 & Panicolin & 76.26 & 0.29 \\
\hline MOL002933 & 5,7,4'-Trihydroxy-8-methoxyflavone & 36.56 & 0.27 \\
\hline MOL002934 & NEOBAICALEIN & 104.34 & 0.44 \\
\hline MOL002937 & DIHYDROOROXYLIN & 66.06 & 0.23 \\
\hline MOL000358 & beta-sitosterol & 36.91 & 0.75 \\
\hline MOL000359 & sitosterol & 36.91 & 0.75 \\
\hline MOL000525 & Norwogonin & 39.4 & 0.21 \\
\hline MOL000552 & 5,2'-Dihydroxy-6,7,8-trimethoxyflavone & 31.71 & 0.35 \\
\hline MOL000073 & ent-Epicatechin & 48.96 & 0.24 \\
\hline MOL001458 & coptisine & 30.67 & 0.86 \\
\hline MOL001490 & bis[(2S)-2-ethylhexyl] benzene-1,2-dicarboxylate & 43.59 & 0.35 \\
\hline MOL001506 & Supraene & 33.55 & 0.42 \\
\hline MOL002879 & Diop & 43.59 & 0.39 \\
\hline MOL002897 & epiberberine & 43.09 & 0.78 \\
\hline MOL008206 & Moslosooflavone & 44.09 & 0.25 \\
\hline MOL010415 & 11,13-Eicosadienoic acid, methyl ester & 39.28 & 0.23 \\
\hline MOL012245 & 5,7,4'-trihydroxy-6-methoxyflavanone & 36.63 & 0.27 \\
\hline MOL012246 & 5,7,4'-trihydroxy-8-methoxyflavanone & 74.24 & 0.26 \\
\hline MOL012266 & rivularin & 37.94 & 0.37 \\
\hline MOL002235 & EUPATIN & 50.8 & 0.41 \\
\hline MOL002251 & Mutatochrome & 48.64 & 0.61 \\
\hline MOL002259 & Physciondiglucoside & 41.65 & 0.63 \\
\hline
\end{tabular}




\begin{tabular}{|c|c|c|c|}
\hline MOL002260 & Procyanidin B-5,3'-O-gallate & 31.99 & 0.32 \\
\hline MOL002268 & rhein & 47.07 & 0.28 \\
\hline MOL002276 & Sennoside E_qt & 50.69 & 0.61 \\
\hline MOL002280 & Torachrysone-8-0-beta-D-(6'-oxayl)-glucoside & 43.02 & 0.74 \\
\hline MOL002281 & Toralactone & 46.46 & 0.24 \\
\hline MOL002288 & Emodin-1-0-beta-D-glucopyranoside & 44.81 & 0.8 \\
\hline MOL002293 & Sennoside D_qt & 61.06 & 0.61 \\
\hline MOL002297 & Daucosterol_qt & 35.89 & 0.7 \\
\hline MOL002303 & palmidin A & 32.45 & 0.65 \\
\hline MOL000471 & aloe-emodin & 83.38 & 0.24 \\
\hline MOL000554 & gallic acid-3-0-(6'-0-galloyl)-glucoside & 30.25 & 0.67 \\
\hline MOL001910 & $\begin{array}{l}\text { 11alpha,12alpha-epoxy-3beta-23-dihydroxy-30-norolean-20-en- } \\
\text { 28,12beta-olide }\end{array}$ & 64.77 & 0.38 \\
\hline MOL001918 & paeoniflorgenone & 87.59 & 0.37 \\
\hline MOL001919 & $\begin{array}{l}\text { (3S,5R,8R,9R,10S,14S)-3,17-dihydroxy-4,4,8,10,14-pentamethyl- } \\
\text { 2,3,5,6,7,9-hexahydro-1 H-cyclopenta[a]phenanthrene-15,16-dione }\end{array}$ & 43.56 & 0.53 \\
\hline MOL001921 & Lactiflorin & 49.12 & 0.8 \\
\hline MOL001924 & paeoniflorin & 53.87 & 0.79 \\
\hline MOL001925 & paeoniflorin_qt & 68.18 & 0.4 \\
\hline MOL001928 & albiflorin_qt & 66.64 & 0.33 \\
\hline MOL001930 & benzoyl paeoniflorin & 31.27 & 0.75 \\
\hline MOL000211 & Mairin & 55.38 & 0.78 \\
\hline MOL000492 & $(+)$-catechin & 54.83 & 0.24 \\
\hline MOL001755 & 24-Ethylcholest-4-en-3-one & 36.08 & 0.76 \\
\hline MOL002670 & Cavidine & 35.64 & 0.81 \\
\hline MOL005030 & gondoic acid & 30.7 & 0.2 \\
\hline MOL000519 & coniferin & 31.11 & 0.32 \\
\hline MOL006936 & 10,13-eicosadienoic & 39.99 & 0.2 \\
\hline MOL006937 & 12,13-epoxy-9-hydroxynonadeca-7,10-dienoic acid & 42.15 & 0.24 \\
\hline MOL006957 & (3S,6S)-3-(benzyl)-6-(4-hydroxybenzyl)piperazine-2,5-quinone & 46.89 & 0.27 \\
\hline
\end{tabular}




\begin{tabular}{|c|c|c|c|}
\hline MOL003578 & Cycloartenol & 38.69 & 0.78 \\
\hline MOL006967 & beta-D-Ribofuranoside, xanthine- 9 & 44.72 & 0.21 \\
\hline MOL013276 & poncirin & 36.55 & 0.74 \\
\hline MOL013277 & Isosinensetin & 51.15 & 0.44 \\
\hline MOL013279 & 5,7,4'-Trimethylapigenin & 39.83 & 0.3 \\
\hline MOL013428 & isosakuranetin-7-rutinoside & 41.24 & 0.72 \\
\hline MOL013430 & Prangenin & 43.6 & 0.29 \\
\hline MOL013433 & prangenin hydrate & 72.63 & 0.29 \\
\hline MOL013435 & poncimarin & 63.62 & 0.35 \\
\hline MOL013436 & isoponcimarin & 63.28 & 0.31 \\
\hline MOL013437 & 6-Methoxy aurapten & 31.24 & 0.3 \\
\hline MOL013440 & citrusin B & 40.8 & 0.71 \\
\hline MOL001798 & neohesperidin_qt & 71.17 & 0.27 \\
\hline MOL001803 & Sinensetin & 50.56 & 0.45 \\
\hline MOL001941 & Ammidin & 34.55 & 0.22 \\
\hline MOL013352 & Obacunone & 43.29 & 0.77 \\
\hline MOL004328 & naringenin & 59.29 & 0.21 \\
\hline MOL005100 & 5,7-dihydroxy-2-(3-hydroxy-4-methoxyphenyl)chroman-4-one & 47.74 & 0.27 \\
\hline MOL005828 & nobiletin & 61.67 & 0.52 \\
\hline MOL005849 & didymin & 38.55 & 0.24 \\
\hline MOL000006 & luteolin & 36.16 & 0.25 \\
\hline MOL007879 & Tetramethoxyluteolin & 43.68 & 0.37 \\
\hline MOL009053 & $\begin{array}{l}\text { 4-[(2S,3R)-5-[(E)-3-hydroxyprop-1-enyl]-7-methoxy-3-methylol-2,3- } \\
\text { dihydrobenzofuran-2-yl]-2-methoxy-phenol }\end{array}$ & 50.76 & 0.39 \\
\hline MOL006129 & 6-methylgingediacetate2 & 48.73 & 0.32 \\
\hline MOL001771 & poriferast-5-en-3beta-ol & 36.91 & 0.75 \\
\hline MOL008698 & Dihydrocapsaicin & 47.07 & 0.19 \\
\hline MOL012921 & stepharine & 31.55 & 0.33 \\
\hline MOL012940 & Spiradine A & 113.52 & 0.61 \\
\hline MOL012946 & zizyphus saponin I_qt & 32.69 & 0.62 \\
\hline
\end{tabular}




\begin{tabular}{|c|c|c|c|}
\hline MOL012961 & jujuboside A_qt & 36.67 & 0.62 \\
\hline MOL012976 & coumestrol & 32.49 & 0.34 \\
\hline MOL012980 & Daechuine S6 & 46.48 & 0.79 \\
\hline MOL012981 & Daechuine S7 & 44.82 & 0.83 \\
\hline MOL012986 & Jujubasaponin V_qt & 36.99 & 0.63 \\
\hline MOL012989 & Jujuboside C_qt & 40.26 & 0.62 \\
\hline MOL012992 & Mauritine D & 89.13 & 0.45 \\
\hline MOL001454 & berberine & 36.86 & 0.78 \\
\hline MOL001522 & (S)-Coclaurine & 42.35 & 0.24 \\
\hline MOL003410 & Ziziphin_qt & 66.95 & 0.62 \\
\hline MOL004350 & Ruvoside_qt & 36.12 & 0.76 \\
\hline MOL005360 & malkangunin & 57.71 & 0.63 \\
\hline MOL000627 & Stepholidine & 33.11 & 0.54 \\
\hline MOL007213 & Nuciferin & 34.43 & 0.4 \\
\hline MOL000783 & Protoporphyrin & 30.86 & 0.56 \\
\hline MOL000787 & Fumarine & 59.26 & 0.83 \\
\hline MOL008034 & $21302-79-4$ & 73.52 & 0.77 \\
\hline MOL008647 & Moupinamide & 86.71 & 0.26 \\
\hline MOL002773 & beta-carotene & 37.18 & 0.58 \\
\hline MOL013357 & $\begin{array}{l}\text { (3S,6R,8S,9S,10R,13R,14S,17R)-17-[(1R,4R)-4-ethyl-1,5- } \\
\text { dimethylhexyl]-10,13-dimethyl-2,3,6,7,8,9,11,12,14,15,16,17- } \\
\text { dodecahydro-1H-cyclopenta[a]phenanthrene-3,6-diol }\end{array}$ & 34.37 & 0.78 \\
\hline
\end{tabular}

\section{Figures}




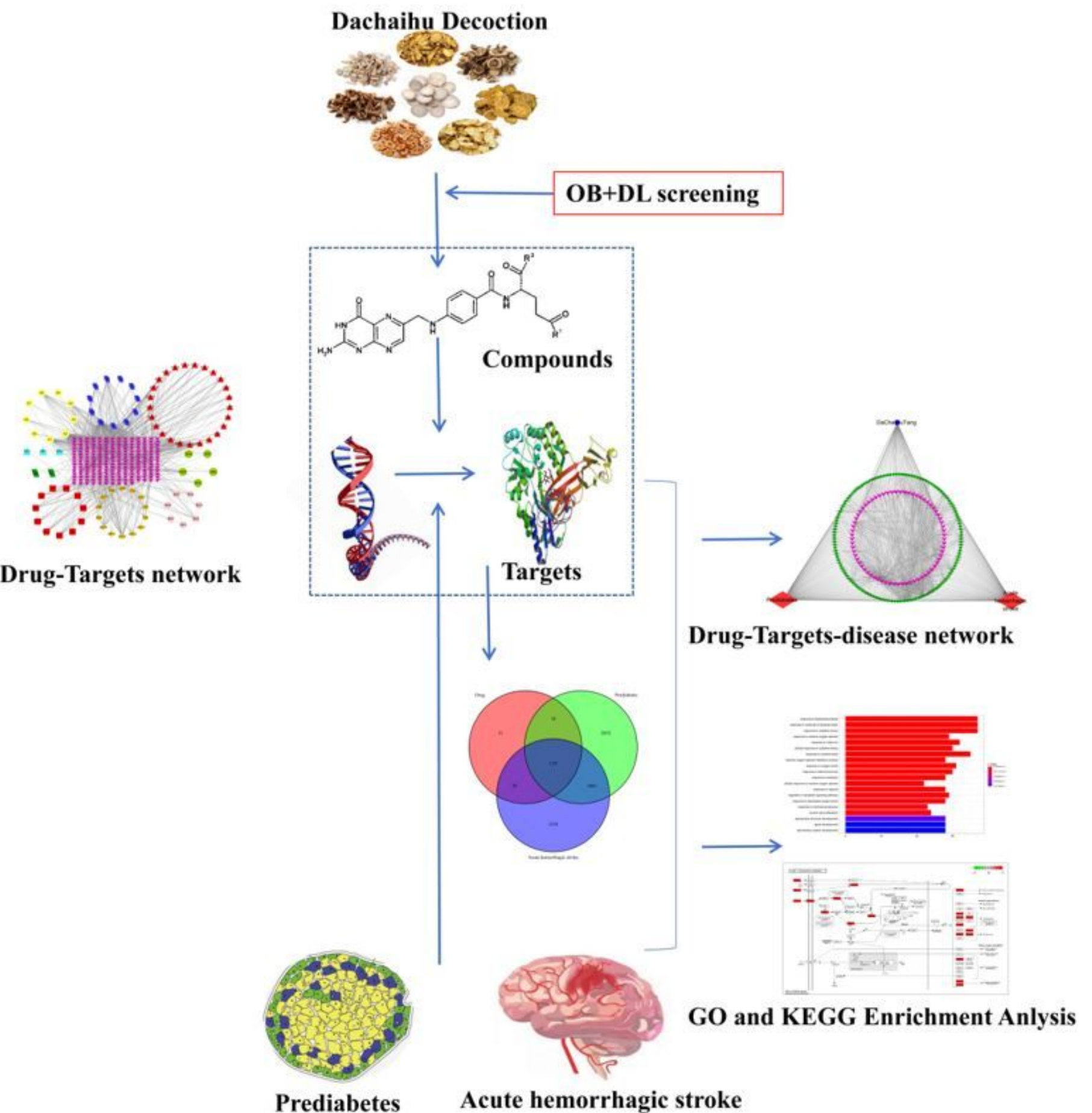

Figure 1

Workflow for Dachaihu Decoction against Acute hemorrhagic stroke based on network pharmacology 


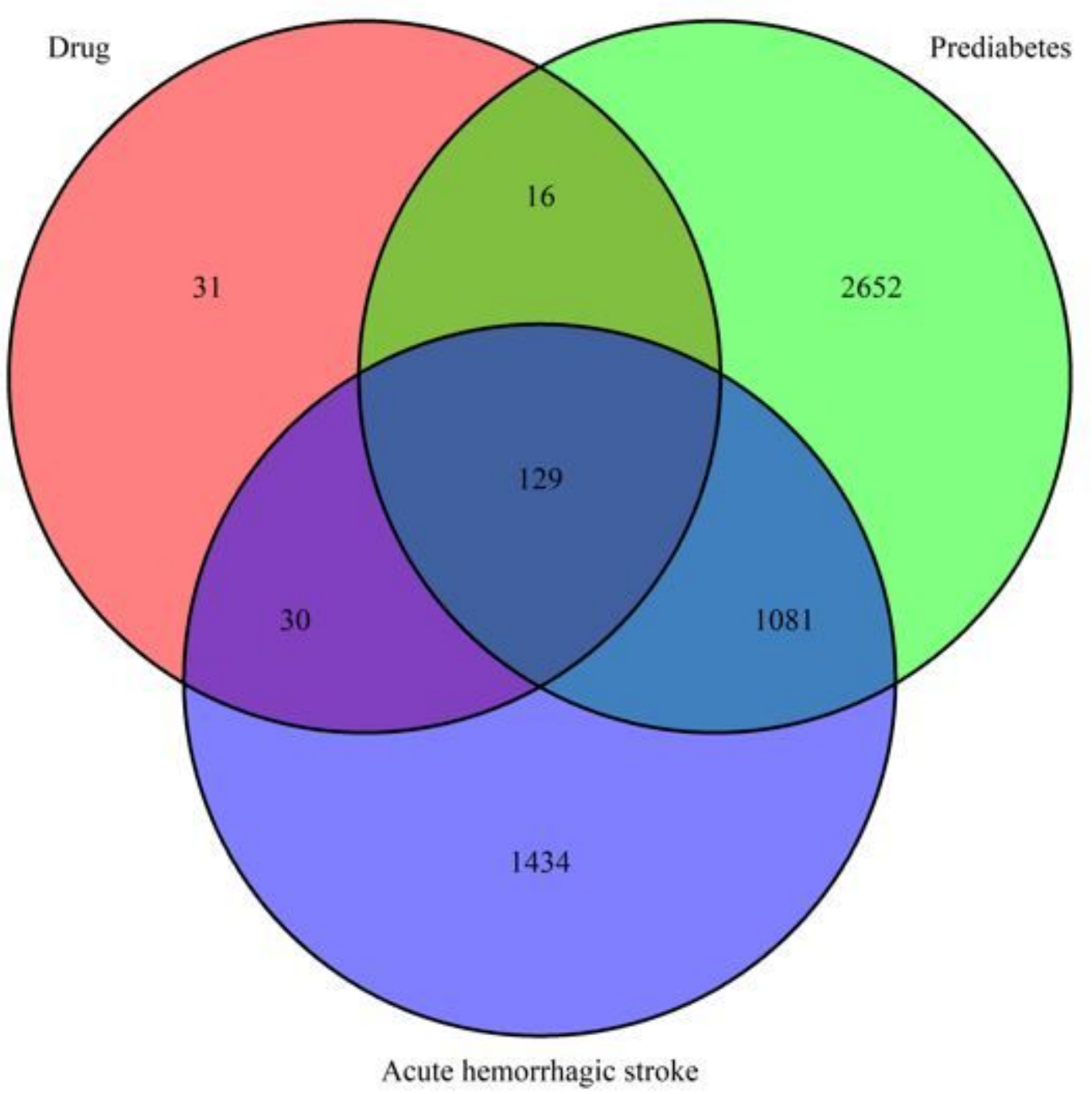

Figure 2

The 129 matching targets of the related targets in DCHD on PD and AHS. 


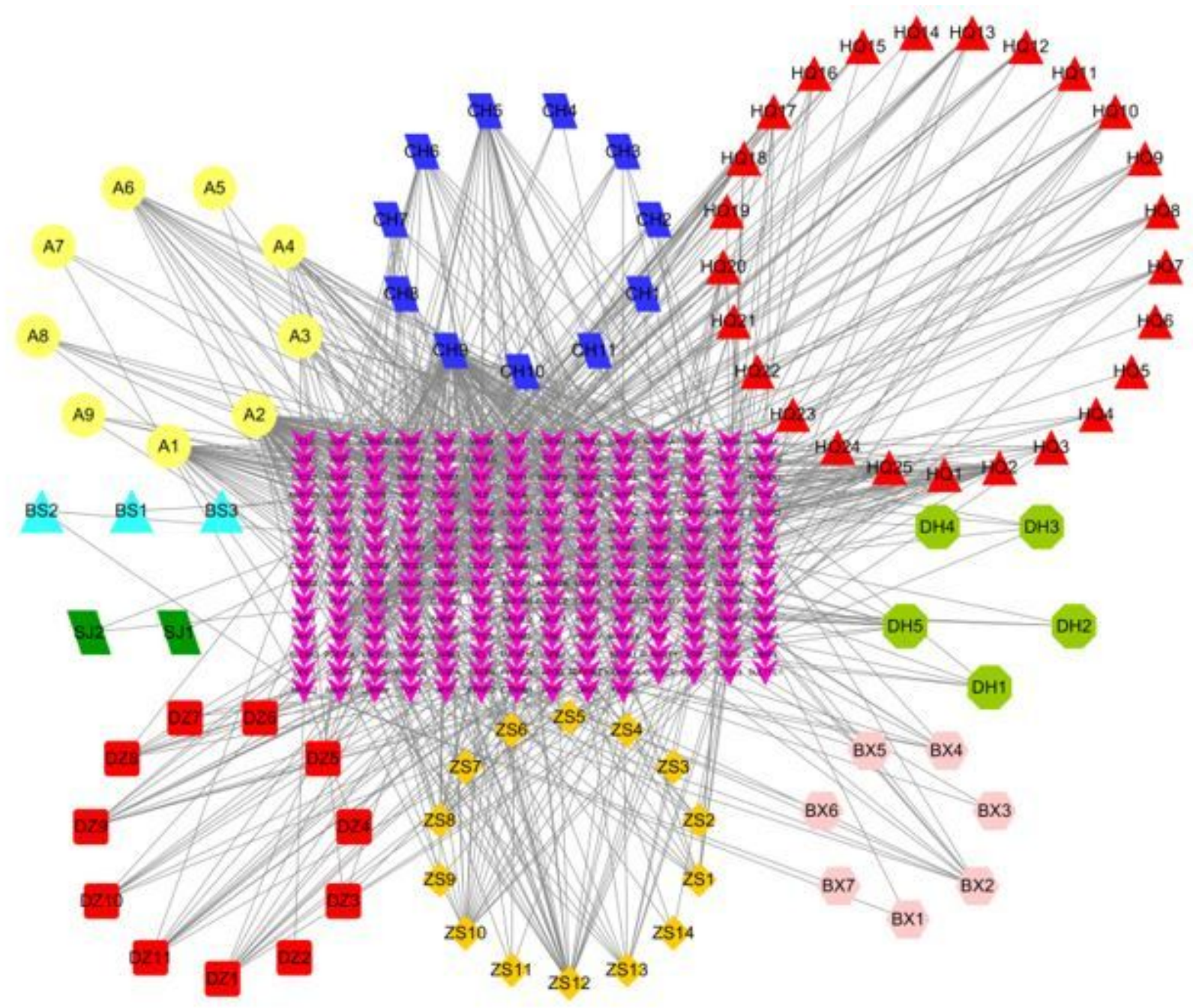

Figure 3

compound target network (Pink V represents compound target network, Yellow Ellipsel represents compound of drug repetition, Blue Parallelogram, Red Triangle, Green Octagon, Light blue Triangle, Light pink Hexagon, Brown Diamond,Dark green Parallelogram and Red Round Rectangle represent compound of $\mathrm{CH}, \mathrm{HQ}, \mathrm{DH}, \mathrm{BS}, \mathrm{BX}, \mathrm{ZS}, \mathrm{SJ}$ and $\mathrm{DZ}$ respectively). 


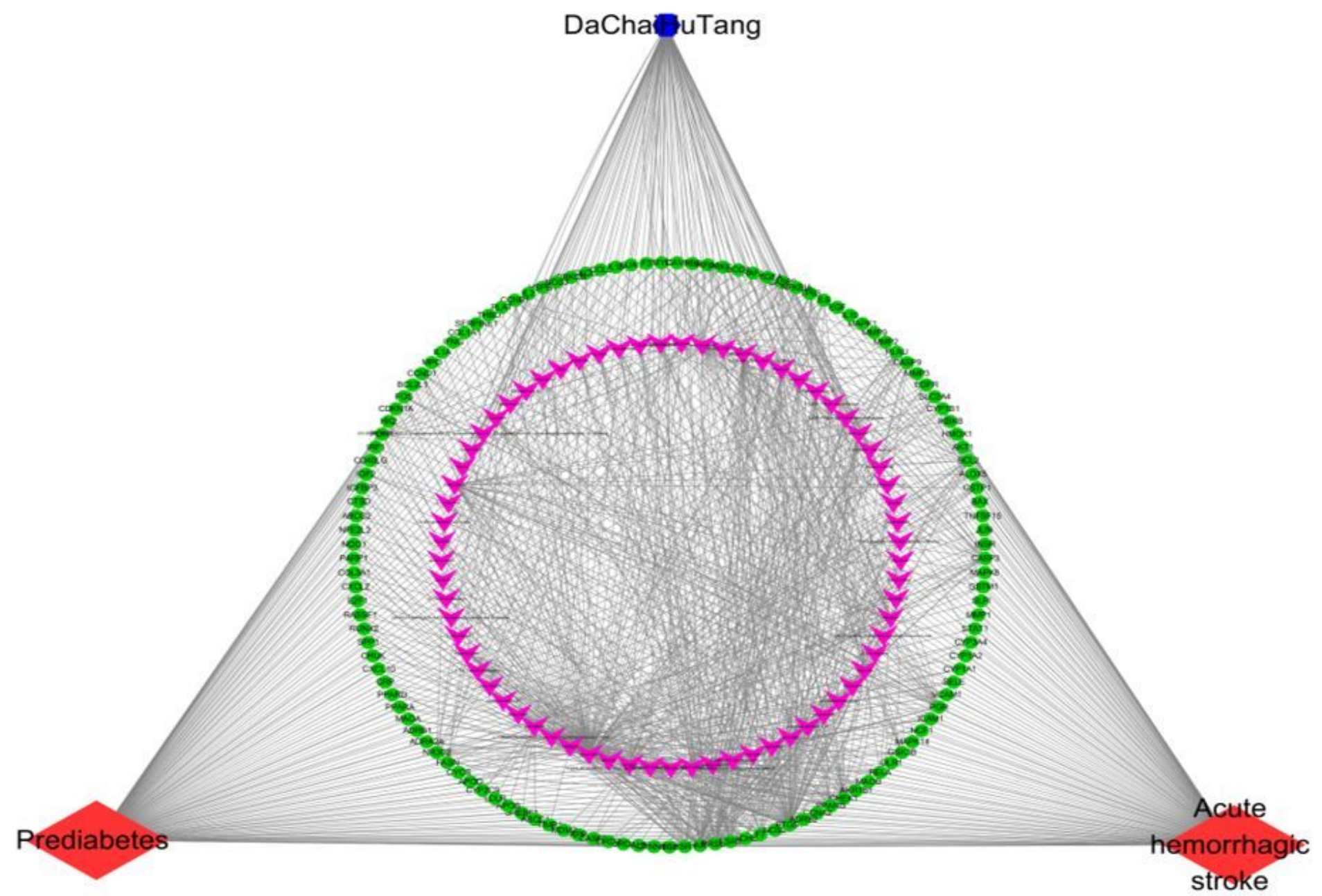

Figure 4

Herb Compounds - disease Target- disease Network (the blue hexagons at the top of the figure represent herbs, the rose red $v$ represent compound, green rounded represents PD and AHS targets, and the bottom red diamond represents disease.) 


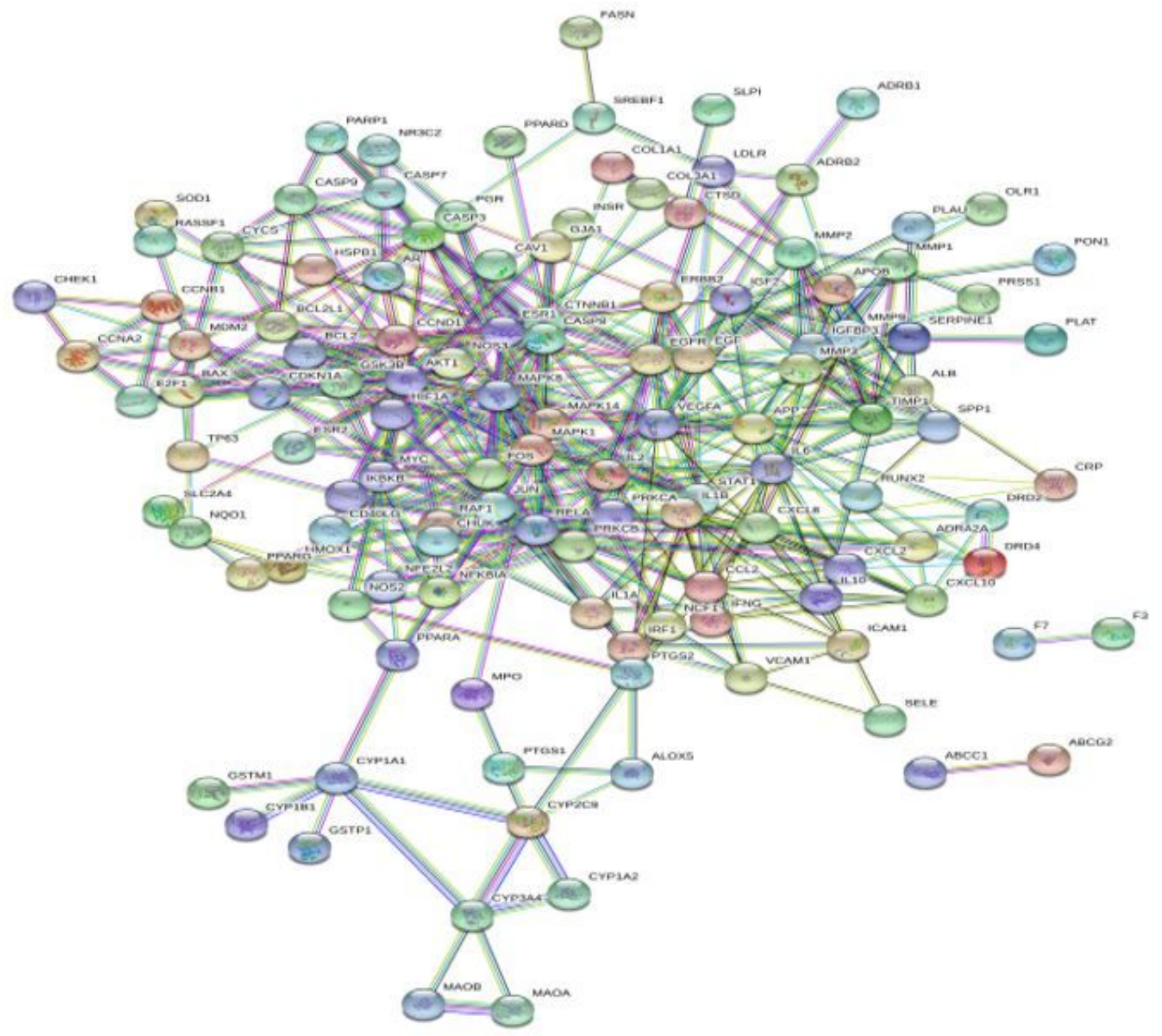

Figure 5

PPI network of 129 nodes and 492 edges established in the String Datebase. 


$$
0
$$




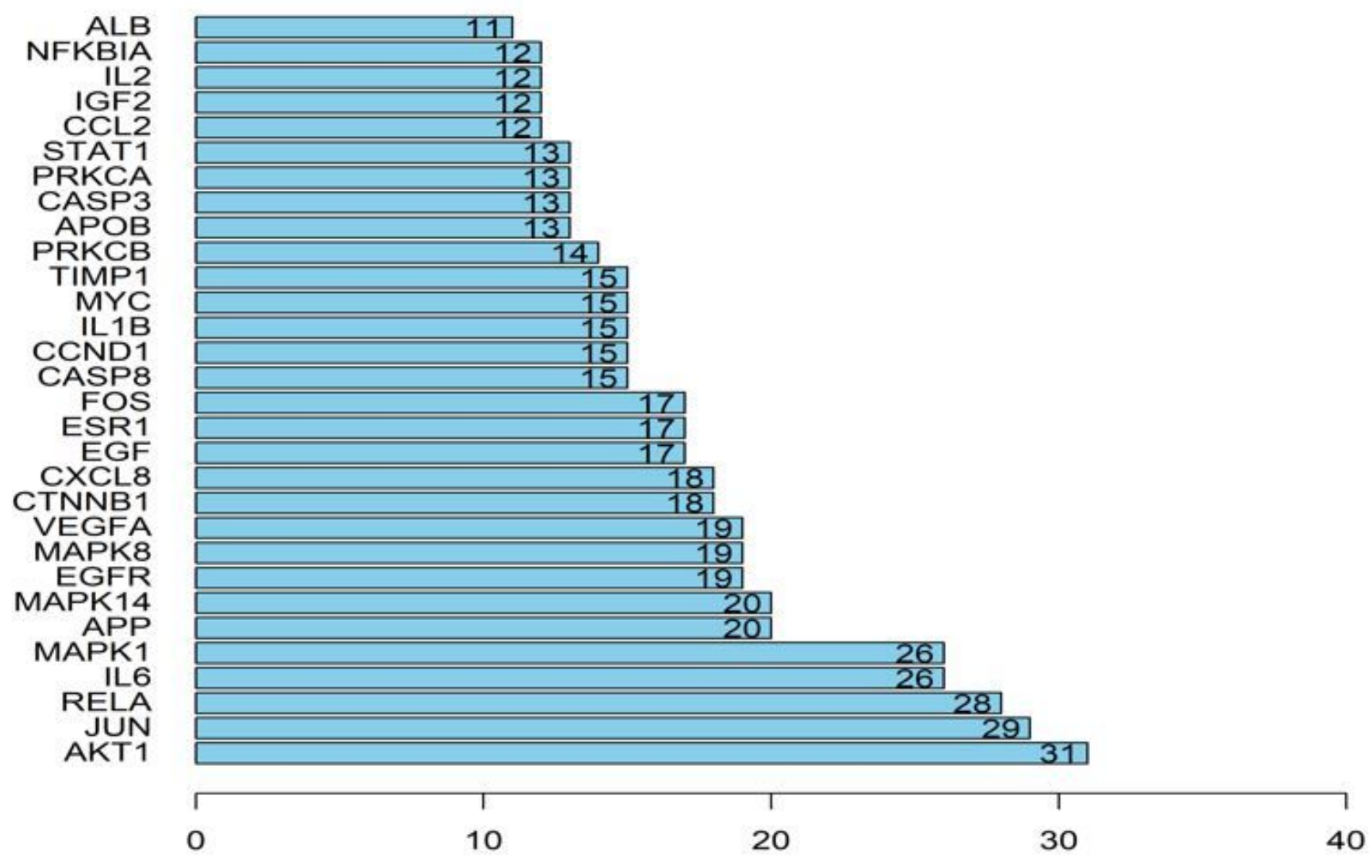

Figure 7

The bar plot of the PPI network. 


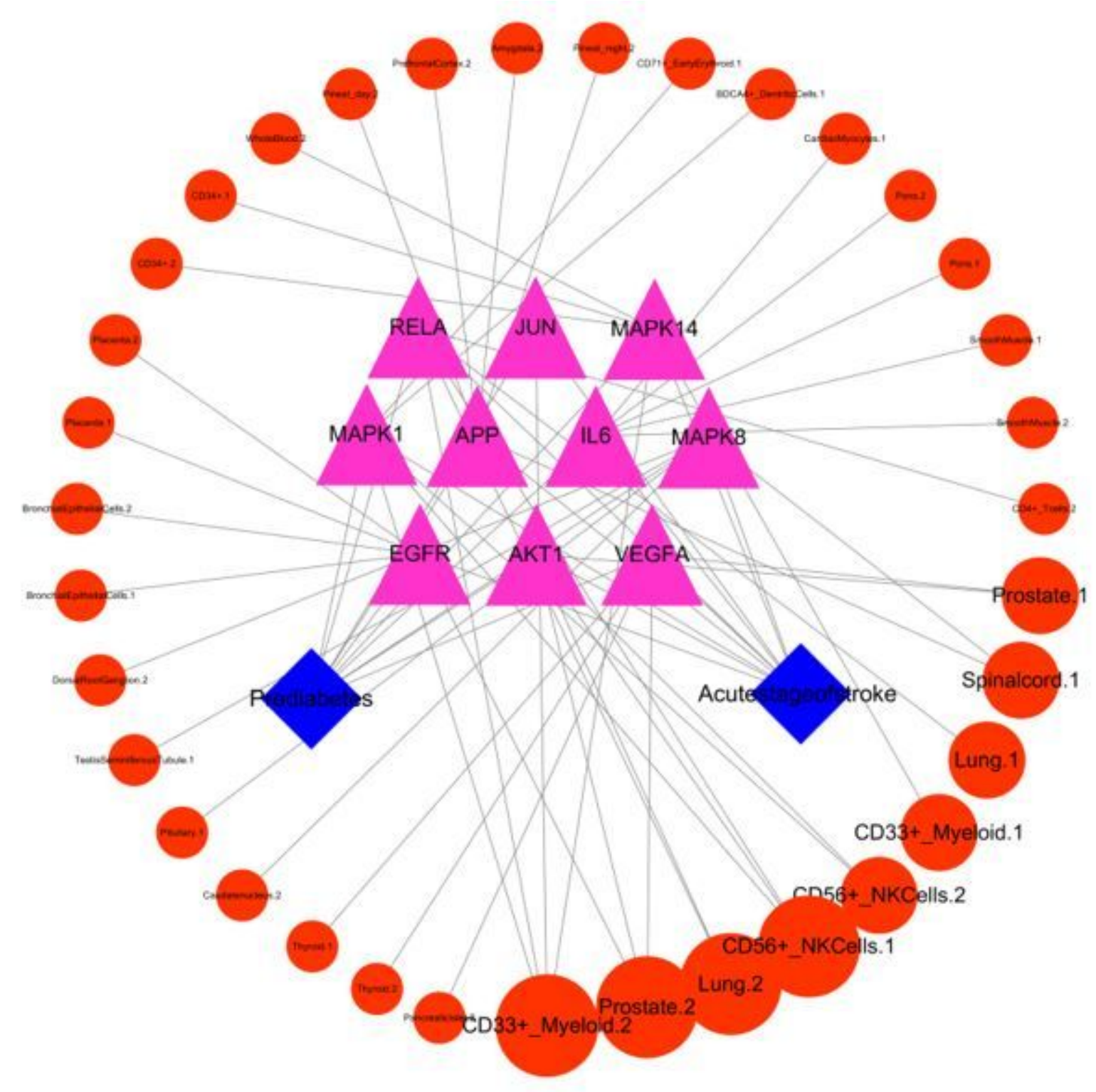

Figure 8

Targets-organs/tissue network of DachaihuTang in treating PD and AHS.(The blue diamond represents the disease, and the pink triangle node is the key target, The red circle node is the organ tissue, and the circle size represents the size of the degree value.) 


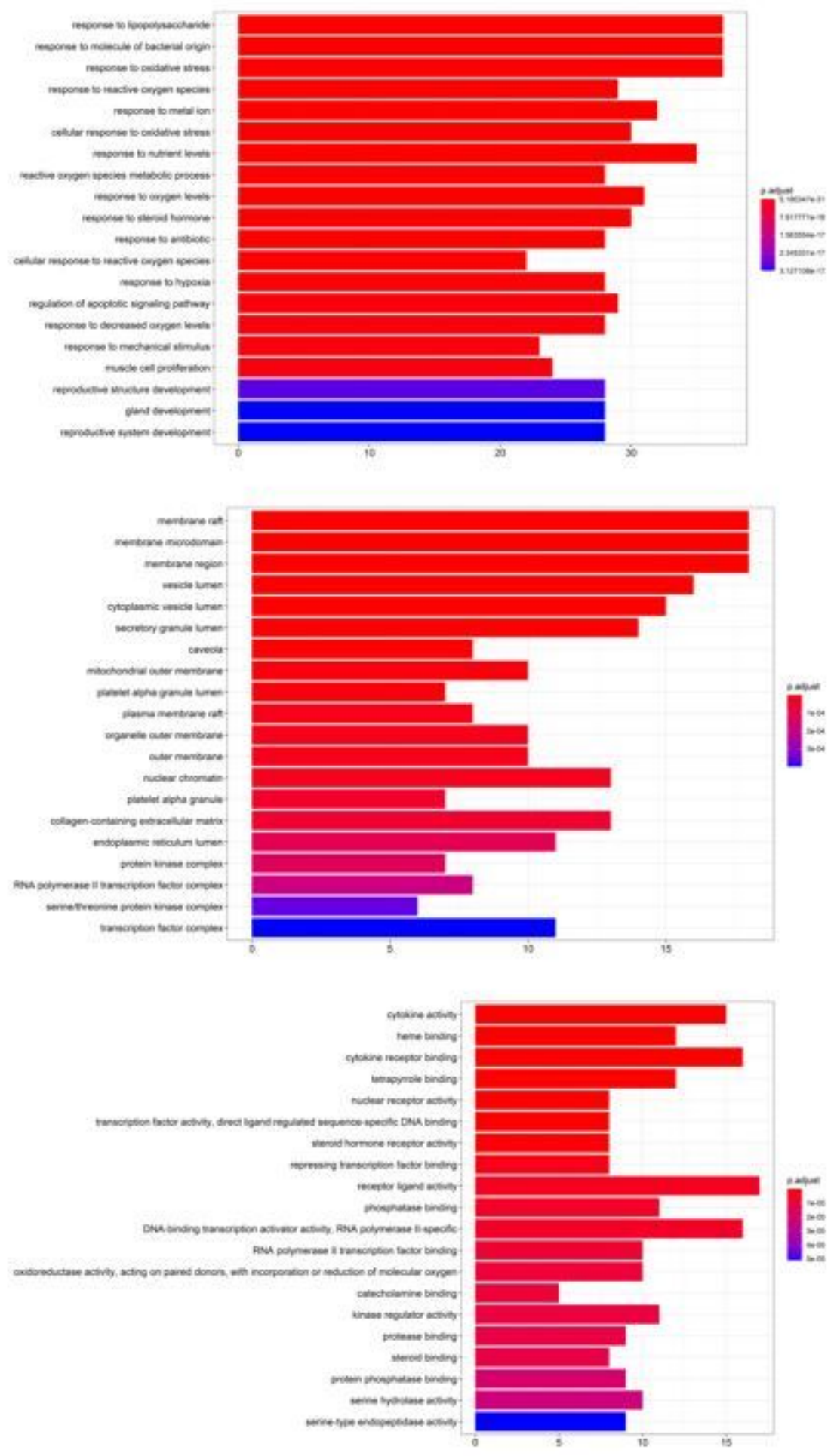

Figure 9

$(\mathrm{a}, \mathrm{b}, \mathrm{c}) \mathrm{GO}$ functional enrichment analysis. a. The top $20 \mathrm{GO}$ enrichments in BP; b. The top $20 \mathrm{GO}$ enrichments in CC; c. The top $20 \mathrm{GO}$ enrichments in MF. The horizontal axis represents the number of genes in the term. The color indicates the p-value. 


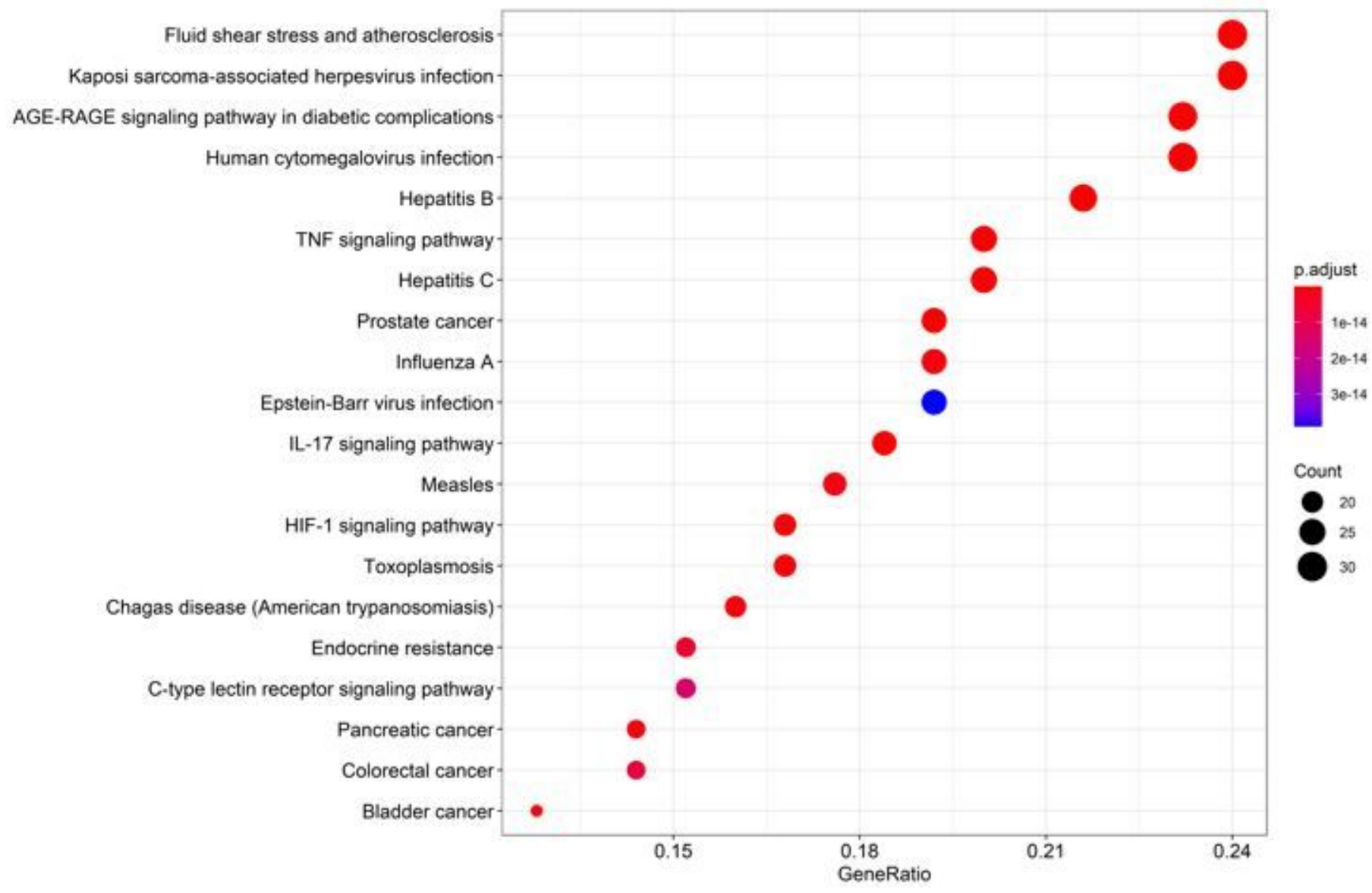

\section{Figure 10}

KEGG enrichment analysis. Each node signaled a GO term, and its size represented the gene number. The color indicates the $p$-value. 


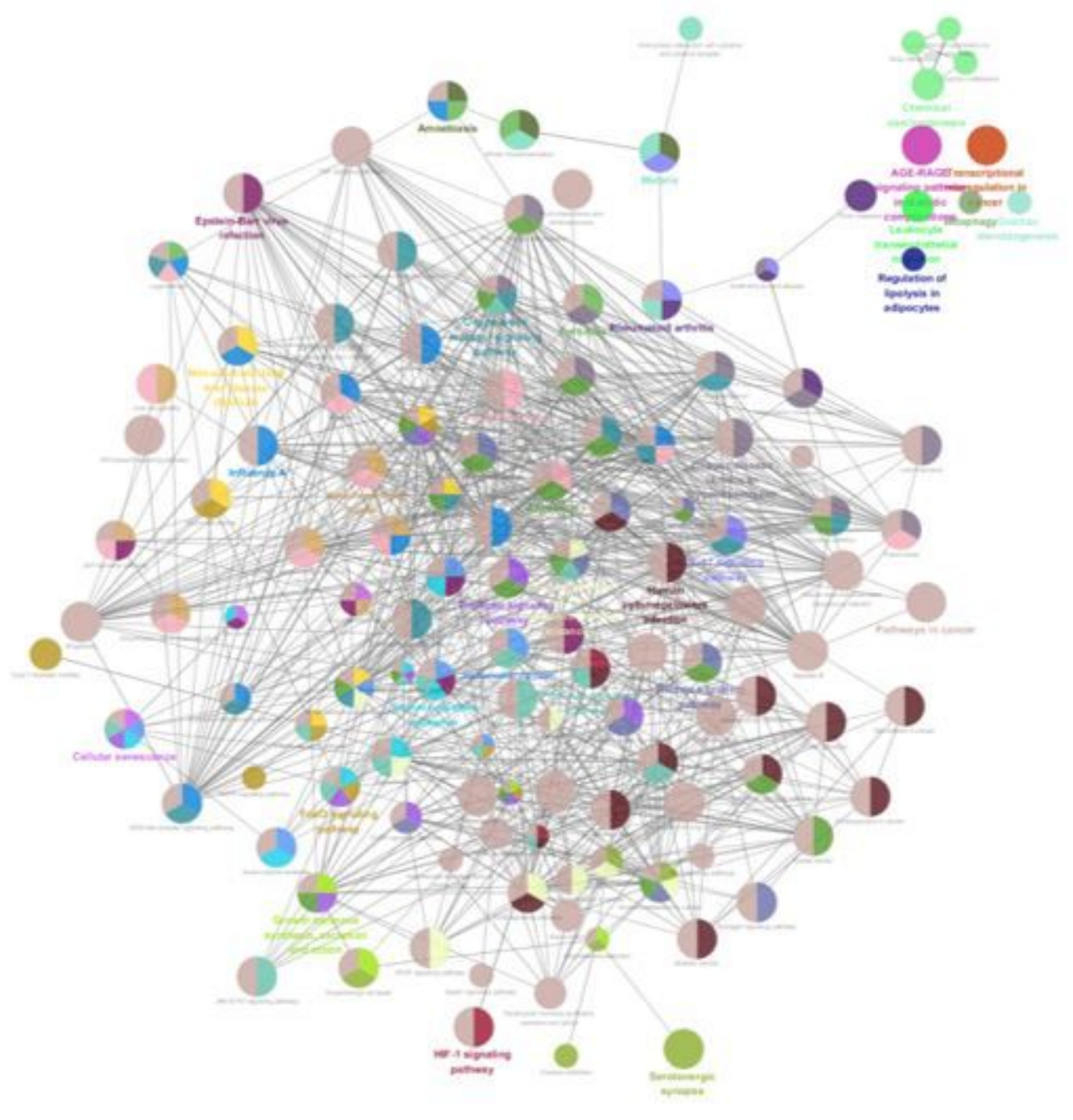

Figure 11

The functionally grouped network of enriched categories was generated for the target genes using ClueGO and CluePedia. Pathway terms were represented as nodes, and the node color represented the grouping information. 